\title{
Influência de fatores sociossanitários na qualidade de vida dos idosos de um município do Sudeste do Brasil
}

\author{
The influence of sociosanitary conditions on the quality of life \\ of the elderly in a municipality in the Southeast of Brazil
}

\author{
Renata Junqueira Pereira ${ }^{1}$ \\ Rosângela Minardi Mitre Cotta ${ }^{2}$ \\ Sylvia do Carmo Castro Franceschini ${ }^{2}$ \\ Rita de Cássia Lanes Ribeiro ${ }^{2}$ \\ Rosana Ferreira Sampaio ${ }^{3}$ \\ Silvia Eloiza Priore ${ }^{2}$ \\ Paulo Roberto Cecon ${ }^{2}$
}

${ }^{1}$ Curso de Nutrição, Universidade Federal do Tocantins. Campus de Palmas. Av. NS 15, ALCNO 14, Bloco de Apoio Logístico e Administrativo I (BALA I), sala 19.

77001-090 Palmas TO. renatajunqueira@uft.edu.br

${ }^{2}$ Universidade Federal de

Viçosa.

${ }^{3}$ Universidade Federal de

Minas Gerais.
Abstract This study sought to evaluate sociosanitary conditions and their impact on the quality of life of the elderly living in the Teixeiras Municipality in the Southeast Region (MG) of Brazil. Two questionnaires were used: one created specifically to characterize the population in terms of sociosanitary conditions, and WHOQOL-bref, to evaluate quality of life. The correlation between the sociosanitary conditions and quality of life scores were analyzed by logistic regression analysis. Among the variables with a strong correlation with quality of life scores the following stand out: use of medication, need of medical care, lack of private health insurance, comorbidities, sleep problems, and retirement. The results of this study indicate that sociosanitary conditions are high risk factors for low quality of life scores in the elderly. This must be taken into consideration to establish health strategies and policies for this age group. Key words Ageing, Quality of life, Health, WHOQOL-bref
Resumo Este estudo teve como objetivo avaliar as condições sociossanitárias e o impacto das delas na qualidade de vida de idosos residentes no município de Teixeiras (MG), na região Sudeste do Brasil. Foram aplicados dois questionários: um criado especificamente para caracterizar a população quanto às características sociossanitárias, $e$ o WHOQOL-bref para avaliar a qualidade de vida. A associação entre as características sociossanitárias e os escores de qualidade de vida foi analisada por meio de análises de regressão logística. Dentre as variáveis mais fortemente associadas aos escores de qualidade de vida destacaramse: utilização de medicamentos, necessidade de cuidados médicos, ausência de cobertura por plano de saúde privado, presença de comorbidades, problemas do sono e aposentadoria. Os resultados do estudo apontam os fatores sociossanitários citados como fatores de risco para baixos escores de qualidade de vida no idoso, o que deve ser considerado ao se estabelecerem estratégias e politicas voltadas a esse grupo populacional.

Palavras-chave Envelhecimento, Qualidade de vida, Saúde, WHOQOL-bref 


\section{Introdução}

O envelhecimento populacional no Brasil iniciouse, sendo a Região Sudeste (considerada a mais desenvolvida) a que apresentou o maior envelhecimento, representando os idosos, atualmente, $7,9 \%$ da população total ${ }^{1}$. De acordo com projeções do Instituto Brasileiro de Geografia e Estatísticas $(\mathrm{IBGE})^{2}$, em 2010 o Brasil terá cerca de 18 milhões de indivíduos com mais de 60 anos, e até 2020 esse número crescerá para 25 milhões.

Atingir a longevidade constituiu um dos maiores êxitos da segunda metade do século XX. O aumento da expectativa de vida é uma conquista social, entretanto almeja-se que esses anos sejam vividos com qualidade. Na maioria dos países do mundo, cada vez mais o envelhecimento da população exigirá um aumento das demandas sanitárias, sociais e econômicas ${ }^{3}$. Nesse sentido, segundo Cotta et al. ${ }^{4}$, o envelhecimento da população, ao mesmo tempo que representa um dos maiores triunfos da humanidade, é um dos maiores desafios.

Segundo Camarano ${ }^{5}$, melhorias nas condições de vida da população idosa diminuiriam os gastos com a saúde e previdenciários, se houvesse uma associação positiva entre maior longevidade e melhores condições de saúde.

Há uma crescente demanda social por alternativas especializadas, destinadas a atender as dificuldades e necessidades do grupo idoso. De acordo com Sousa et al. ${ }^{6}$, a obtenção de dados para a determinação e a caracterização da qualidade de vida e bem-estar dos idosos é fundamental para permitir um envelhecimento bemsucedido. Para esses autores, um envelhecimento bem-sucedido é acompanhado de qualidade de vida e bem-estar, devendo ser fomentado ao longo da vida dos indivíduos.

A Organização Mundial da Saúde (OMS) integra vários conceitos, definindo a qualidade de vida como a percepção pelo indivíduo de sua posição na vida, no contexto da cultura, bem como no sistema de valores em que vive, assim como em relação a seus objetivos, expectativas, padrões e preocupações. Assim, a $\mathrm{OMS}^{7}$ propõe que, para avaliar a qualidade de vida, devam ser estudadas seis áreas: saúde física, estado psicológico, nível de independência, relações sociais, meio ambiente e espiritualidade.

Qualidade de vida é um conceito que vem adquirindo importância na sociedade em geral e é um constructo muito apreciado, sobretudo em relação aos adultos e idosos, constituindo um referencial no âmbito da gerontologia e no pla- nejamento de políticas sociossanitárias para a população idosa ${ }^{8}$.

Assim, reveste-se de grande importância o estudo do perfil sociossanitário das populações idosas para se identificarem os principais indicadores de bem-estar na velhice, ou seja, longevidade, saúde biológica e mental, capacidade funcional, presença de enfermidades crônicas não transmissíveis, utilização de medicamentos, papel social, situação socioeconômica, renda, escolaridade, continuidade de papéis ocupacionais, relações familiares, produtividade, atividade física e hábitos de vida saudáveis ${ }^{9}$.

O conceito de qualidade de vida tem sido considerado na literatura como um conceito básico no campo da atenção ao idoso e é um dos principais indicadores a ser considerado ao se avaliar a eficácia das ações prestadas a esse grupo populacional ${ }^{10}$.

Nesse sentido, este estudo tem como objetivo avaliar as características sociossanitárias e suas possíveis influências na qualidade de vida de idosos residentes no município de Teixeiras (MG), na região Sudeste do Brasil.

\section{Métodos}

\section{Caracterização do município em estudo}

O município de Teixeiras integra a microrregião de Viçosa e a mesorregião da Zona da Mata do Estado de Minas Gerais, situado na Região Sudeste do Brasil. Segundo o censo demográfico realizado em 2000, Teixeiras possuía uma população total de 11.149 habitantes, distribuída em 6.949 habitantes na zona urbana $(62,33 \%)$ e 4.200 $(37,67 \%)$ na zona rural, apresentando uma taxa de urbanização de $62,33 \%$ e uma taxa média de crescimento anual de 1,23\%. Em relação ao contingente de idosos, dos 11.149 habitantes, 1.374 tinham 60 anos ou mais, o que representa 12,32\% da população total ${ }^{2,11}$.

\section{Sujeitos}

Foram incluídos no estudo indivíduos com idade igual ou superior a $60 \operatorname{anos}^{12}$.

Em um primeiro momento do estudo, realizou-se um cadastro prévio de todos os indivíduos com idade igual ou superior a 60 anos, até a data prevista para o início do estudo. Esse cadastro foi realizado com o auxílio dos Agentes Comunitários de Saúde (ACS) que compõem as equipes do Programa de Saúde da Família (PSF) do 
município. Por meio deste cadastro obteve-se a relação de todos os idosos residentes em Teixeiras, já que o PSF cobre $100 \%$ da população do município. O PSF é responsável pela porta de entrada no sistema sanitário nos municípios brasileiros, sendo as atividades executadas por equipe multiprofissional em nível de atenção primária.

Como resultado deste levantamento, tem-se que a população idosa do município é composta por um total de 1.478 indivíduos. Destes, a maioria $(965-65,3 \%)$ se encontra residindo na zona urbana; 775 (52,4\%) são do sexo feminino; 964 $(65,2 \%)$ são usuários do PSF; $101(6,8 \%)$ se encontram acamados, $56(3,8 \%)$ inconscientes e 20 $(1,4 \%)$ institucionalizados.

\section{Seleção da amostra}

Realizou-se amostragem aleatória, estratificada por sexo, utilização dos serviços do PSF e residir na microárea de saúde. Devido às características da entrevista, com o auxílio dos profissionais de saúde do PSF, foram excluídos os indivíduos que se encontravam acamados, inconscientes, institucionalizados e aqueles que não se encontravam em condições para responder ao questionário, totalizando 1.301 idosos.

Aplicados os critérios de exclusão, foram calculados 15\% dos indivíduos cadastrados em cada uma das dezoito microáreas de saúde, respeitando a proporção por sexo e usuários e não usuários do PSF na microárea. Esse procedimento foi realizado considerando-se que cada microárea de saúde representa uma região geográfica do município, garantindo assim que a amostra fosse representativa do município.

Após a estratificação e a determinação do número de indivíduos para cada microárea realizou-se o sorteio da amostra, que foi composta por de 211 idosos, representando, portanto, $14,28 \%$ da população de idosos do município.

\section{Desenho do estudo}

Trata-se de um estudo observacional de corte transversal. Os questionários foram aplicados por um único entrevistador, devidamente treinado, em visitas domiciliares. Realizou-se previamente um estudo piloto em população com características semelhantes às de Teixeiras, porém em município distinto do estudado.

Um dos questionários, especificamente criado para este estudo, coletou informações como: sexo, idade, escolaridade, renda, zona de residência, situação conjugal, apoio familiar, condições da habitação, núcleo familiar, atividade profissional, cobertura previdenciária, prática de atividade física, morbidades autorreferidas, utilização de medicamentos, sono, tabagismo, ingestão de bebidas alcoólicas e utilização de serviços de saúde, objetivando caracterizar os idosos quanto às variáveis sociossanitárias.

Para avaliar a qualidade de vida foi utilizado o instrumento proposto pela OMS, o Word Health Organization Quality of Life Instrument Bref (WHOQOL-bref). O WHOQOL-bref é uma versão reduzida do Word Health Organization Quality of Life Instrument 100 (WHOQOL-100), sendo composto por 24 questões referentes a cada faceta do WHOQOL-100, mais duas questões de avaliação global de qualidade de vida. O WHOQOL-bref analisa quatro domínios: capacidade física, bem-estar psicológico, relações sociais e meio ambiente ${ }^{13}$.

A versão abreviada demonstrou características satisfatórias quanto à confiabilidade testereteste e à validade discriminante, apresentando como principal vantagem o rápido preenchimento. O questionário WHOQOL já foi traduzido para mais de vinte idiomas. No Brasil, foi analisado quanto às validades interna, discriminante, concorrente e de conteúdo ${ }^{14}$.

A versão brasileira do WHOQOL-bref, traduzida e validada pelo grupo de estudos em qualidade de vida da OMS no Brasil, foi utilizada neste estudo.

Os escores finais de cada domínio são calculados por uma sintaxe que considera as respostas de cada questão que compõe o domínio, resultando em escores finais numa escala de 4 a 20, comparáveis ao WHOQOL 100, que podem ser transformados em escala de 0 a 100.

O instrumento em questão é autoaplicável, mas neste estudo optou-se pela aplicação pelo pesquisador, dada a dificuldade de leitura, problemas visuais e o analfabetismo, comuns em idosos.

O protocolo de pesquisa foi aprovado pelo Comitê de Ética em Pesquisas com Seres Humanos da Universidade Federal de Viçosa, Minas Gerais, Brasil. Todos os indivíduos que aceitaram participar do estudo assinaram um Termo de Consentimento Livre Esclarecido.

\section{Análise dos dados}

Inicialmente foi calculada a Razão de Chances (Odds Ratio - OR) e o intervalo de confiança (IC 95\%) para cada variável sociossanitária em relação a cada um dos domínios da qualidade de 
vida. Os escores de cada um dos domínios da qualidade de vida e as variáveis sociossanitárias foram dicotomizados em presença ou ausência de risco para baixos escores de qualidade de vida.

Para cada domínio da qualidade de vida, os indivíduos foram divididos em "abaixo ou igual" e "acima" da mediana do grupo, sendo considerados com baixa qualidade de vida os indivíduos "abaixo ou igual" à mediana. Esta divisão de grupos foi realizada dado o fato de não existirem pontos de corte estabelecidos para escores de qualidade de vida. As medianas encontradas para os diferentes domínios do WHOQOL-bref foram 16,0 para os domínios global, físico e psicológico; 17,3 para o social e 14,5 para o ambiental.

Em relação às variáveis sociossanitárias, para a idade foi calculada a mediana do grupo (70 anos), e os indivíduos foram divididos em "abaixo ou igual" e "acima" da mediana, sendo considerados em risco para uma baixa qualidade de vida os indivíduos acima da mediana de idade, conforme a literatura ${ }^{15-18}$. Para as demais variáveis sociossanitárias, ser do sexo feminino, residir na zona rural, não utilizar os serviços do PSF, não possuir infraestrutura básica adequada no domicílio, dormir em quarto individual, ter banheiro localizado fora do domicílio, não exercer atividade profissional, ser aposentado, não praticar atividade física, possuir alguma enfermidade, apresentar número de enfermidades autorreferidas acima da mediana do grupo (uma enfermidade), utilizar algum medicamento, fazer uso de um número de medicamentos acima da mediana do grupo (três medicamentos), não dormir bem, fumar, ingerir bebidas alcoólicas, não ter plano de saúde privado, ter consultado o médico nos últimos seis meses, ter sofrido alguma internação no último ano, viver sem esposo(a) ou companheiro(a), não ter apoio da família, ter menos de quatro anos de escolaridade (analfabeto funcional), ter renda $<1$ salário mínimo (baixa renda) e morar só foram considerados riscos para escores de qualidade de vida "abaixo ou igual" à mediana, nos domínios do WHOQOL-bref.

As variáveis que apresentaram OR com significância $<$ a $20 \%$ de probabilidade $(\mathrm{p}<0,20)$ foram inseridas em modelos de regressão logística múltipla para identificar os fatores associados a baixos escores de qualidade de vida. Foram construídos cinco modelos de regressão logística, cada um com um domínio como variável dependente. Para entrar na análise, as variáveis sociossanitárias foram dicotomizadas em valores 0 e 1 , sendo atribuído o valor 1 para os fatores de risco.
Foram calculados intervalos de confiança de 95\% para as OR. A significância foi fixada em 5\% de probabilidade. O modelo ajustado foi verificado usando o Teste de Hosmer e Lemeshow.

Para as análises foi utilizado o software Statistical Package for the Social Sciences (SPSS) for Windows, versão $10.0^{19}$.

\section{Resultados}

Em relação à caracterização sociossanitária, dos 211 idosos estudados, 51,7\% são do sexo feminino e o restante masculino. A idade do grupo variou entre 60 a 93 anos com média de $71( \pm 8,1)$ e mediana de 70 anos. Mais da metade dos idosos reside na zona urbana (66\%).

Entre os idosos estudados, $71,1 \%$ são analfabetos funcionais, ou seja, possuem menos de quatro anos de estudo formal ${ }^{11}$, enquanto apenas $28,9 \%$ são considerados alfabetizados. Em relação à renda mensal, 69,7\% recebiam valor igual ou inferior a um salário mínimo.

Descrevendo a situação conjugal, 37,5\% vivem sem companheiro. A maioria dos idosos estudados relatou receber apoio emocional da família (97,6\%). A análise da composição do domicílio mostra que coexistem diversos tipos de arranjos familiares e cerca de 15\% dos entrevistados moram sozinhos. Em relação ao compartilhamento do quarto, $64 \%$ o fazem com pelo menos um familiar e 36\% têm quarto individual. Em 10,5\% dos domicílios, o banheiro tem localização externa ao domicílio.

Mais da metade dos idosos estudados $(61,1 \%)$ não mais exercem atividade profissional, mas 38,9\% ainda continuam desempenhando atividades laborais (serviços domésticos, autônomos e com vínculo empregatício). A situação previdenciária mostra que $73 \%$ dos idosos estudados são aposentados, 15,2\% estão desempregados, isto é, não trabalham e não recebem benefícios previdenciários, e 11,8\% não são aposentados, ou seja, ainda trabalham e não recebem benefícios previdenciários.

No que se refere à saúde, $63 \%(\mathrm{n}=133)$ dos entrevistados são usuários do PSF. Em relação à assistência à saúde privada, 29,9\% possuem plano de saúde.

O percentual de usuários do PSF que possuía plano de saúde privado foi de $18,8 \%$. Em relação aos não usuários do PSF, 51,5\% não possuíam plano privado.

O perfil dos idosos que não possuem plano de saúde privado $(\mathrm{n}=148)$, é de renda mensal 
menor ou igual a um SM (77\%); idade acima de 70 anos (46\%); portadores de pelo menos uma enfermidade crônica e usuários de pelo menos um medicamento $(73,6 \%)$; usuários de consultas médicas no mínimo uma vez nos últimos seis meses $(76,3 \%)$.

Em relação aos usuários do PSF $(n=133)$, $73,7 \%$ possuem renda menor ou igual a um salário mínimo; $45,9 \%$ possuem idade acima de 70 anos; $85,7 \%$ consultaram pelo menos uma vez nos últimos seis meses; $81,9 \%$ possuem pelo menos uma enfermidade crônica; e 83,4\% utilizam pelo menos um medicamento.

Quanto às demais características de saúde, $55 \%$ praticam atividade física regularmente; $76,8 \%$ relataram a presença de pelo menos uma enfermidade crônica; 76,8\% utilizam algum medicamento; $39,8 \%$ relatam problemas do sono; 19,4\% fumam; 22,8\% fazem uso de bebidas alcoólicas; $76,3 \%$ estiveram em consulta médica nos últimos seis meses; e $23,7 \%$ sofreram pelo menos uma internação hospitalar no último ano.

Os resultados significativos $(\mathrm{p}<0,20)$ das razões de chances para cada domínio da qualidade de vida com as variáveis sociossanitárias estão demonstrados na Tabela 1.

Destaca-se que a idade (corte mediano) não se mostrou fator de risco para baixos escores de qualidade de vida, por isso optou-se por não controlar a idade nas análises de regressão.

Foram construídos cinco modelos de regressão logística múltipla para identificar os fatores de risco para uma qualidade de vida "abaixo ou igual" à mediana. As variáveis mostradas na Tabela 1 foram inseridas na análise para cada domínio. Nas Tabelas 2 a 5 são apresentados os modelos ajustados para cada domínio da qualidade de vida.

Observa-se que os fatores significativamente associados a escores abaixo ou iguais à mediana do grupo para o domínio global do WHOQOLbref foram não possuir plano de saúde privado e ter consultado o médico pelo menos uma vez nos últimos seis meses (Tabela 2). A chance de estar abaixo ou igual à mediana de qualidade de vida global é 2,43 vezes maior, quando não se possui plano de saúde privado; e 2,44 vezes maior, quando se consultou o médico nos últimos seis meses. O modelo ajustado explica $8,2 \%$ do domínio global.

Para o domínio físico do WHOQOL-bref, os fatores significativamente associados a escores abaixo ou iguais à mediana foram consultar o médico pelo menos uma vez nos últimos seis meses, possuir mais de uma enfermidade autorreferida, não dormir bem e ser aposentado ( Ta- bela 3). A chance de estar abaixo ou igual à mediana do grupo para o domínio físico foi 2,90 vezes maior para os indivíduos que consultaram nos últimos seis meses, 2,35 vezes maior para os que possuíam mais de uma enfermidade autorreferida, 2,40 vezes maior para os que não dormiam bem ou tinham insônia e 2,57 vezes maior para os que eram aposentados. O modelo ajustado explica, portanto, 19,6\% do domínio físico.

No que se refere ao domínio social do WHOQOL-bref, os fatores significativamente associados a escores abaixo ou iguais à mediana foram a localização do banheiro fora do domicílio, não possuir plano de saúde privado e fumar (Tabela 4). A chance de estar abaixo ou igual à mediana do grupo para o domínio social foi 2,77 vezes maior quando o banheiro era localizado fora do domicílio e 2,19 vezes maior quando não se possuía plano de saúde privado. O hábito de fumar reduziu a chance de estar abaixo ou igual à mediana para o domínio social. O modelo ajustado explica, neste caso, 8,4\% do domínio social.

No domínio psicológico, os fatores significativamente associados a escores abaixo ou iguais à mediana foram não dormir bem e utilizar pelo menos um medicamento (Tabela 5), com chance 1,92 vez maior quando não se dorme bem ou se tem insônia e 1,99 vez maior quando se utiliza pelo menos um medicamento. Observa-se que o modelo ajustado explica pouco $(5,5 \%)$ o domínio psicológico.

Para o domínio ambiental, os fatores significativamente associados a baixos escores foram consultar pelo menos uma vez nos últimos seis meses, ser do sexo feminino, não possuir plano de saúde privado e ser aposentado (Tabela 5). A chance de estar abaixo ou igual à mediana do grupo para o domínio ambiental foi 2,37 vezes maior para os que consultaram nos últimos seis meses; 2,95 vezes maior, para as mulheres; 2,27 vezes maior, na ausência de plano de saúde privado, e 2,81 vezes maior para os aposentados. O modelo ajustado explicou $11,1 \%$ do domínio ambiental.

\section{Discussão}

Os resultados de nosso estudo mostram a influência que têm as condições de saúde na qualidade de vida dos idosos. Em todos os domínios, pelo menos duas variáveis sanitárias estiveram associadas a menores escores de qualidade de vida.

Estudo realizado pelo IMSERSO ${ }^{20}$ também mostrou ser a saúde a principal preocupação dos idosos, que apontaram a saúde e a independência 
como aspectos mais importantes para a qualidade de vida. Por sua vez, nossos resultados apontaram a ausência de plano de saúde privado como característica de saúde que mais se destacou, estando associada a baixos escores de qualidade de vida nos domínios global, social e ambiental.

Tabela 1. Odds ratio das variáveis sociossanitárias com os domínios da qualidade de vida - Teixeiras (Minas Gerais, Brasil), 2004.

\begin{tabular}{|c|c|c|c|c|}
\hline Domínio & Fator & OR & IC $(95 \%)$ & $\mathrm{p}$ \\
\hline Global & Não possuir plano de saúde privado & 2,86 & $1,43-5,72$ & $0,003^{*}$ \\
\hline$n=168$ & Consultar nos últimos seis meses & 2,33 & $1,13-4,81$ & $0,024^{*}$ \\
\hline \multirow[t]{6}{*}{$\mathrm{N}=43$} & Baixa renda & 2,16 & $1,08-4,32$ & $0,031^{*}$ \\
\hline & $\mathrm{N}^{\circ}$ enfermidades acima da mediana & 2,18 & $1,03-4,61$ & $0,034^{*}$ \\
\hline & Zona residência rural & 2,26 & $1,02-5,03$ & $0,035^{\star}$ \\
\hline & Não utilizar o PSF & 0,53 & $0,27-1,06$ & 0,074 \\
\hline & Utilizar algum medicamento & 1,83 & $0,87-3,83$ & 0,114 \\
\hline & Banheiro fora do domicílio & 2,77 & $0,62-12,34$ & 0,132 \\
\hline Físico & Consultar nos últimos seis meses & 4,31 & $2,12-8,74$ & $0,000^{*}$ \\
\hline $\mathrm{n}=110$ & $\mathrm{~N}^{\circ}$ enfermidades acima da mediana & 3,39 & $1,88-6,09$ & $0,000^{*}$ \\
\hline \multirow[t]{10}{*}{$N=101$} & Possuir alguma enfermidade & 3,21 & $1,62-6,36$ & $0,001^{\star}$ \\
\hline & Não dormir bem & 2,70 & $1,52-4,80$ & $0,001^{*}$ \\
\hline & Não trabalhar & 2,39 & $1,35-4,21$ & $0,002^{*}$ \\
\hline & Utilizar algum medicamento & 2,53 & $1,30-4,93$ & $0,005^{\star}$ \\
\hline & Fumar & 0,40 & $0,19-0,81$ & $0,010^{*}$ \\
\hline & Não utilizar o PSF & 0,53 & $0,30--0,93$ & $0,028^{*}$ \\
\hline & Ser aposentado & 1,73 & $0,94-3,21$ & 0,076 \\
\hline & Não praticar atividade física & 1,52 & $0,88-2,63$ & 0,129 \\
\hline & Ingerir bebidas alcoólicas & 0,60 & $0,31-1,17$ & 0,136 \\
\hline & $\mathrm{N}^{\circ}$ medicamentos acima da mediana & 1,50 & $0,83-2,71$ & 0,171 \\
\hline Psicológico & Não dormir bem & 2,55 & $1,27-3,98$ & $0,005^{*}$ \\
\hline $\mathrm{n}=113$ & Utilizar algum medicamento & 2,44 & $1,26-4,72$ & $0,007^{*}$ \\
\hline \multirow[t]{9}{*}{$\mathrm{N}=98$} & Sexo feminino & 1,94 & $1,12-3,36$ & $0,017^{\star}$ \\
\hline & Possuir alguma enfermidade & 2,18 & $1,13-4,19$ & $0,018^{*}$ \\
\hline & Não trabalhar & 1,74 & $0,99-3,04$ & $0,050^{*}$ \\
\hline & $\mathrm{N}^{\circ}$ enfermidades acima da mediana & 1,69 & $0,96-2,97$ & 0,063 \\
\hline & Ser aposentado & 0,58 & $0,31-1,08$ & 0,087 \\
\hline & Baixa renda & 1,60 & $0,89-2,90$ & 0,113 \\
\hline & Consultar nos últimos seis meses & 1,65 & $0,87-3,13$ & 0,121 \\
\hline & Fumar & 0,61 & $0,31-1,22$ & 0,168 \\
\hline & Internação no último ano & 1,57 & $0,82-3,00$ & 0,168 \\
\hline Social & Banheiro fora do domicílio & 2,97 & $1,18-7,44$ & $0,017^{*}$ \\
\hline $\mathrm{n}=84$ & Não possuir plano saúde privado & 2,01 & $1,06-3,79$ & $0,027^{\star}$ \\
\hline \multirow[t]{6}{*}{$\mathrm{N}=127$} & Zona residência rural & 1,59 & $0,89-2,83$ & 0,115 \\
\hline & Fumar & 0,56 & $0,26-1,17$ & 0,119 \\
\hline & Ser aposentado & 1,62 & $0,85-3,09$ & 0,134 \\
\hline & $\mathrm{N}^{\circ}$ enfermidades acima da mediana & 1,50 & $0,85-2,64$ & 0,154 \\
\hline & Quarto individual & 1,49 & $0,84-2,65$ & 0,166 \\
\hline & Não dormir bem & 1,45 & $0,82-2,55$ & 0,191 \\
\hline Ambiental & Consultar nos últimos seis meses & 2,37 & $1,22-4,57$ & $0,009^{*}$ \\
\hline $\mathrm{n}=110$ & Sexo feminino & 1,87 & $1,08-3,23$ & $0,024^{*}$ \\
\hline \multirow[t]{7}{*}{$\mathrm{N}=101$} & Baixa renda & 1,94 & $1,07-3,53$ & $0,027^{\star}$ \\
\hline & Não possuir plano saúde privado & 1,86 & $1,02-3,39$ & $0,039^{*}$ \\
\hline & Não trabalhar & 1,58 & $0,90-2,76$ & 0,104 \\
\hline & $\mathrm{N}^{\circ}$ enfermidades acima da mediana & 1,58 & $0,90-2,76$ & 0,105 \\
\hline & Banheiro fora do domicílio & 2,12 & $0,82-5,43$ & 0,107 \\
\hline & Não dormir bem & 1,51 & $0,86-2,64$ & 0,142 \\
\hline & Ser aposentado & 1,57 & $0,85-2,90$ & 0,143 \\
\hline
\end{tabular}

n: Número de idosos abaixo da mediana de qualidade de vida; N: Número de idosos acima da mediana de qualidade de vida. 
Tabela 2. Fatores associados a baixos escores de qualidade de vida no domínio global do WHOQOL-bref Teixeiras (Minas Gerais, Brasil), 2004.

\begin{tabular}{lcccccr}
\hline & Odds ratio & IC $(\mathbf{9 5} \%)$ & $\mathbf{p}$ & $\hat{\boldsymbol{\beta}}$ & $\mathbf{R}^{219}$ & $\mathbf{H L}^{\S}$ \\
\hline Domínio global & & & & $-0,298$ & & \\
$\quad$ Não possuir plano de saúde privado & 2,43 & $1,16-5,08$ & $0,018^{*}$ & 0,890 & & \\
Consultar nos últimos seis meses & 2,44 & $1,14-5,19$ & $0,021^{*}$ & 0,892 & 0,082 & 0,934 \\
Zona residência rural & 1,78 & $0,77-4,14$ & $0,177^{\mathrm{NS}}$ & 0,581 & & \\
Baixa renda & 1,55 & $0,73-3,29$ & $0,253^{\mathrm{NS}}$ & 0,439 & & \\
\hline
\end{tabular}

§: Teste de Hosmer and Lemeshow goodness-of-fit; ": Significativo a 5\% de probabilidade; Ns: Fatores não significativos foram incluídos para ajuste do modelo.

Tabela 3. Fatores associados a baixos escores de qualidade de vida no domínio físico do WHOQOL-bref Teixeiras (Minas Gerais, Brasil), 2004.

\begin{tabular}{|c|c|c|c|c|c|c|}
\hline & Odds ratio & IC $(95 \%)$ & $\mathbf{p}$ & $\hat{\boldsymbol{\beta}}$ & $\mathbf{R}^{2} 19$ & $\mathrm{HL}^{\S}$ \\
\hline Domínio físico & & & & $-2,344$ & & \\
\hline Consultar nos últimos seis meses & 2,90 & $1,24-6,82$ & $0,014^{*}$ & 1,067 & & \\
\hline $\mathrm{N}^{\circ}$ enfermidades acima da mediana & 2,35 & $1,17-4,72$ & $0,016^{*}$ & 0,857 & 0,196 & 0,599 \\
\hline Não dormir bem & 2,40 & $1,25-4,62$ & $0,009^{*}$ & 0,876 & & \\
\hline Ser aposentado & 2,57 & $1,26-5,25$ & $0,010^{*}$ & 0,945 & & \\
\hline Possuir alguma enfermidade & 1,14 & $0,47-2,73$ & $0,765^{\mathrm{NS}}$ & 0,133 & & \\
\hline Não trabalhar & 1,49 & $0,79-2,82$ & $0,216^{\mathrm{NS}}$ & 0,401 & & \\
\hline Não utilizar o PSF & 0,75 & $0,39-1,45$ & $0,389^{\mathrm{NS}}$ & $-0,290$ & & \\
\hline
\end{tabular}

§: Teste de Hosmer and Lemeshow goodness-of-fit; ${ }^{*}$ : Significativo a 5\% de probabilidade; ${ }^{\text {Ns}}$ : Fatores não significativos foram incluídos para ajuste do modelo.

Tabela 4. Fatores associados a baixos escores de qualidade de vida no domínio social do WHOQOL-bref Teixeiras (Minas Gerais, Brasil), 2004.

\begin{tabular}{lccccrr}
\hline & Odds ratio & IC (95\%) & $\mathbf{p}$ & $\hat{\boldsymbol{\beta}}$ & $\mathbf{R}^{219}$ & $\mathbf{H L}^{\S}$ \\
\hline Domínio social & & & & $-1,582$ & & \\
$\quad$ Banheiro fora do domicílio & 2,77 & $1,06-7,22$ & $0,036^{*}$ & 1,020 & & \\
$\quad$ Não possuir plano saúde privado & 2,19 & $1,12-4,27$ & $0,022^{*}$ & 0,784 & 0,084 & 0,826 \\
Fumar & 0,45 & $0,20-0,99$ & $0,047^{*}$ & $-0,794$ & & \\
Ser aposentado & 1,80 & $0,91-3,56$ & $0,089^{\mathrm{NS}}$ & 0,590 & & \\
Não dormir bem & 1,65 & $0,91-3,01$ & $0,098^{\mathrm{NS}}$ & 0,505 & & \\
\hline
\end{tabular}

§: Teste de Hosmer and Lemeshow goodness-of-fit; ; : Significativo a 5\% de probabilidade; NS: Fatores não significativos foram incluídos para ajuste do modelo.

Tabela 5. Fatores associados a baixos escores de qualidade de vida nos domínios psicológico e ambiental do WHOQOL-bref - Teixeiras (Minas Gerais, Brasil), 2004.

\begin{tabular}{lcccccc}
\hline & Odds ratio & IC (95\%) & $\mathbf{p}$ & $\hat{\boldsymbol{\beta}}$ & $\mathbf{R}^{219}$ & $\mathbf{H L}^{\S}$ \\
\hline Domínio psicológico & & & & $-0,642$ & & \\
$\quad$ Não dormir bem & 1,92 & $1,06-3,47$ & $0,031^{*}$ & 0,654 & 0,055 & 0,900 \\
$\quad$ Utilizar algum medicamento & 1,99 & $1,00-3,96$ & $0,049^{*}$ & 0,690 & & \\
Domínio ambiental & & & & $-2,454$ \\
$\quad$ Consultar nos últimos seis meses & 2,37 & $1,19-4,71$ & $0,014^{*}$ & 0,862 & & \\
$\quad$ Sexo feminino & 2,95 & $1,52-5,73$ & $0,001^{*}$ & 1,082 & \multirow{2}{*}{0,111} & 0,786 \\
$\quad$ Não possuir plano de saúde privado & 2,27 & $1,19-4,33$ & $0,012^{*}$ & 0,821 & & \\
$\quad$ Ser aposentado & 2,81 & $1,35-5,84$ & $0,006^{*}$ & 1,034 & & \\
\hline
\end{tabular}

§: Teste de Hosmer and Lemeshow goodness-of-fit; " : Significativo a 5\% de probabilidade. 
Estudos destacam que os serviços privados de saúde tendem, pela seleção adversa, a negligenciar os idosos e os portadores de doenças crônicas, excluindo esses indivíduos do acesso a serviços de saúde pelo alto custo das mensalidades ${ }^{21,22}$. Talvez esta seja uma hipótese explicativa para o fato de a ausência de plano privado de saúde estar influenciando a qualidade de vida da população estudada, ou seja, em geral os que não possuem plano de saúde privado são mais doentes, possuem fragilidades financeiras e necessitam de maior atenção à saúde, fato observado em nosso estudo, em que os idosos sem plano de saúde privado, na maioria, são de baixa renda, necessitam de cuidados à saúde, possuem enfermidades crônicas e utilizam medicamentos continuamente.

Outra característica de saúde relevante foi ter consultado pelo menos uma vez nos últimos seis meses, estando associada a baixos escores de qualidade de vida nos domínios global, físico e ambiental, ou seja, em nosso estudo, os idosos acometidos por enfermidades e/ou suas complicações percebem pior sua qualidade de vida. Nossos resultados se assemelham ao estudo realizado por Castellón e Pino ${ }^{8}$, em que os idosos associam estreitamente um bom estado de saúde a uma boa qualidade de vida.

Um aspecto que também merece destaque é o fato de estar aposentado se associar significativamente a piores escores de qualidade de vida nos domínios físico e ambiental. Esse achado vai ao encontro das teorias de que a perda do papel social e o declínio da funcionalidade, que normalmente ocorrem com a aposentadoria, interferem marcantemente na qualidade de vida ${ }^{23}$.

De forma geral, os escores de qualidade de vida subjetiva são maiores para os homens do que para as mulheres, talvez porque o envelhecimento seja percebido mais negativamente pelas mulheres ${ }^{8}$. Porém, a razão desta diferença ainda é obscura, havendo somente hipóteses ${ }^{17,24}$. Em nosso estudo, ser do sexo feminino mostrou-se associado significativamente a baixos escores apenas no domínio ambiental, em que os indivíduos fazem uma análise do meio ambiente em que estão inseridos, podendo as mulheres mostrarem-se mais insatisfeitas do que os homens, considerando-se que a preocupação da mulher com a casa, a aparência e condições do local onde vive é mais evidente ${ }^{25}$.

É importante ressaltar um resultado inusitado encontrado, em que o hábito de fumar reduz a chance de baixos escores no domínio social. Como destaca Hulse ${ }^{26}$, o uso de tabaco raramente é escondido pelo paciente idoso. Porém, depois de toda a vida fumando, as pessoas mais velhas podem ser mais relutantes em deixar o vício do que grupos de idade mais jovens, pois os idosos consideram que os danos do cigarro já ocorreram em seu organismo. A família também pode não apoiar a mudança, por considerar que o ato de fumar é um dos poucos prazeres de que o idoso usufrui. Além disso, estudo mostrou ser o tabaco frequentemente utilizado pelo idoso como forma de refúgio da solidão, de aumentar o ânimo e a autoestima, de ajudar no sono, no relaxamento e até no alívio da dor ${ }^{27}$. No entanto, a literatura comprova que deixar de fumar representa, para o idoso, melhorias significativas nas condições de saúde, inclusive uma provável diminuição do risco de perda da capacidade cognitiva e Doença de Alzheimer ${ }^{28}$.

É consenso que os problemas do sono podem causar dificuldades no desempenho cognitivo, distúrbios do humor, problemas emocionais, mau desempenho no trabalho e em atividades de lazer, além de prejuízo do bem-estar físico e mental ${ }^{29}$. Neste estudo, evidenciou-se que não dormir bem esteve significativamente associado a baixos escores nos domínios físico e psicológico, mostrando a importância do sono no bem-estar dos idosos. Igualmente, estudo desenvolvido por Manocchia et al. ${ }^{30}$ mostrou diminuição significativa nos escores de qualidade de vida à medida que se aumentavam os níveis de problemas do sono.

Ainda no domínio psicológico, encontrou-se associação entre utilizar medicamentos e baixos escores de qualidade de vida. Atualmente, o uso de medicamentos pelos idosos tem gerado preocupação quanto aos gastos excessivos e aos possíveis efeitos, muitas vezes, indesejáveis.

No Brasil, estima-se que $23 \%$ da população consumam $60 \%$ da produção de medicamentos, e que 64,5 milhões de pessoas ( $\pm 35 \%$ da população) em condições de pobreza não tenham acesso a esses remédios; e ainda, no grupo dos idosos, as mulheres mais velhas, com maior renda familiar e com mais sintomas, são as que mais utilizam os medicamentos prescritos ${ }^{31}$.

De acordo com Hulse ${ }^{26}$, as mudanças fisiológicas que acompanham o processo de envelhecimento influenciam as concentrações séricas de medicamentos e seu metabolismo, de forma que a polifarmácia e a interação entre drogas podem influenciar negativamente a capacidade funcional, bem como a habilidade psicomotora e cognitiva dos idosos - o que aumenta o risco de acidentes, ferimentos, isolamento e, finalmente, institucionalização. 
No domínio social, uma característica que esteve significativamente associada a baixos escores e que chama a atenção foi a localização do banheiro fora do domicílio. Talvez isso possa ser explicado pelo fato de ser mais difícil para o idoso se locomover até o banheiro fora de casa, principalmente durante a noite; além de poder causar certo constrangimento em relação às pessoas estranhas ao domicílio, fato observado durante as visitas domiciliares realizadas.

É importante destacar que neste estudo a idade não foi fator de risco significativo para escores de qualidade de vida abaixo da mediana, embora a literatura confirme que com o aumento da idade haja uma redução dos escores de qualidade de vida ${ }^{15-18}$. Uma possível explicação seria que, em nosso estudo, a comparação foi realizada entre indivíduos de uma mesma faixa etária (idosos de 60 a 100 anos), enquanto outros trabalhos realizam comparações entre indivíduos das faixas etárias mais jovens e os idosos.

Jakobsson et al. ${ }^{17}$ ressaltam que, apesar do declínio da qualidade de vida com o aumento da idade, nem todos os idosos a relatam baixa; com o envelhecimento, há um declínio nos aspectos físicos da qualidade de vida, porém pode não haver mudanças, ou apenas discretas alterações, nos aspectos mentais, podendo assim serem explicadas as diferenças no número de pessoas idosas que experimentam uma qualidade de vida boa ou aceitável. Covinsky et al. ${ }^{32}$, em estudo de população idosa ( 80 a 101 anos), verificaram que mais de $51 \%$ relataram boa ou muito boa qualidade de vida.

Além disso, renda e escolaridade não estiveram significativamente associadas aos escores de qualidade de vida em nosso estudo, embora a literatura considere esses fatores como influentes $^{33}$. De acordo com Jakobsson et al. ${ }^{17}$, a renda se reduz acentuadamente quando ocorre a aposentadoria, e os fatores socioeconômicos podem influenciar a qualidade de vida, tendo sido encontradas associações entre melhores condições socioeconômicas e melhores escores de qualidade de vida. Sherbourne et al. ${ }^{24}$ também verificaram que problemas financeiros reduziam o bem estar dos idosos. Sprangers et al. ${ }^{34}$ mostraram que o baixo nível de escolaridade esteve relacionado a baixos níveis de qualidade de vida.

Cabe também ressaltar que o fato de ser usuário ou não dos serviços do PSF não se associou significativamente aos escores de qualidade de vida em nenhum dos domínios do WHOQOL- bref. No entanto, as equipes do PSF podem direcionar seu planejamento e suas ações tendo as características sociossanitárias dos idosos como referência, principalmente aquelas que interferem na qualidade de vida do idoso, buscando melhorias da qualidade de vida e do cuidado a esta população.

É importante destacar ainda que este estudo é de corte transversal, permitindo apenas mostrar associações e não uma relação de causalidade entre as variáveis. No entanto, é necessário pesquisar os fatores associados tanto a baixos quanto a altos escores de qualidade de vida, com o objetivo de melhor entender a qualidade de vida, e ser capaz de propor melhorias (ou, pelo menos, manter) a qualidade de vida e o cuidado ao idoso ${ }^{17}$.

Convém notar também que, com um total de 5.564 municípios, o Brasil hoje é composto, em sua grande maioria, por municípios de pequeno porte, registrando uma porcentagem de 73,1 (4.075 municípios) com população de até 20 mil habitantes. Esses dados estabelecem os pequenos municípios dentre os principais protagonistas na implementação e na gestão do Sistema Único de Saúde (SUS), não podendo ser excluídos da lista de prioridades ${ }^{35}$.

Outro fator relevante é que nesses municípios de pequeno porte a maioria dos estabelecimentos de saúde são públicos, gerando assim maior responsabilização dos gestores municipais em trabalhar as políticas do SUS ${ }^{35}$.

Conhecer a qualidade de vida do idoso e os fatores que contribuem para uma redução ou aumento da qualidade de vida torna-se imprescindível aos provedores de cuidados em saúde, gestores e políticos, para que sejam capazes de melhorar a vida diária e a qualidade de vida da população idosa; além de fator essencial a ser considerado quando se propõem ações e políticas de saúde e qualidade de vida para esta população.

Assim, no planejamento de ações em saúde e qualidade de vida para esta população idosa, atenção especial deve ser dada às características sanitárias que mostraram-se fatores de risco para uma baixa qualidade de vida, como a utilização de medicamentos, a necessidade de cuidados médicos, a ausência de plano de saúde privado, a presença de comorbidades e problemas do sono. Além de se considerar, em especial, os indivíduos aposentados, buscando devolver papéis ocupacionais e manter a capacidade funcional desses indivíduos. 


\section{Colaboradores}

RJ Pereira participou da idealização, do delineamento do objeto de estudo, desenho da metodologia, coleta e análise dos dados e redação do artigo; RMM Cotta, da idealização, do delineamento do objeto de estudo, desenho da metodologia, coordenação, orientação e acompanhamento do trabalho de campo, redação e revisão do artigo; SCC Franceschini, do planejamento, orientação técnica e acompanhamento do trabalho; RCL Ribeiro, RF Sampaio e SE Priori, da redação do artigo e sua revisão crítica; PR Cecon, da orientação técnica e estatística do trabalho.

\section{Agradecimentos}

Ao apoio da Prefeitura Teixeiras e do Programa Saúde da Família. Dedicamos este estudo aos profissionais do PSF e aos idosos que aceitaram participar, pois sem eles este trabalho não seria possível. Agradecimentos especiais a José Antônio Brilhante e Danielle Góes pelos valiosos ensinamentos estatísticos. Este trabalho foi apoiado parcialmente pelo Ministério da Saúde do Brasil.

\section{Referências}

1. Brasil. Ministério da Saúde. Saúde planeja atenção a idosos do Nordeste [documento na internet]. Brasília: Ministério da Saúde; 2008. [acessado 2008 mar 05]. Disponível em: http://portal.saude.gov.br/portal/ aplicacoes/noticias/noticias_detalhe.cfm?co_seq_ noticia $=28391$

2. Instituto Brasileiro de Geografia e Estatística (IBGE). Dados preliminares do censo 2000 [documento na internet]. [Internet]. Brasília: IBGE; 2000. [acessado 2008 fev 20]. Disponível em: http://www.ibge. gov.br.

3. Pereira RJ, Cotta RMM, Priore SE. Políticas sobre envelhecimento e saúde no mundo. O Mundo da Saúde 2005; 29(4):475-483.

4. Cotta RMM, Suárez-Varela MM, Filho JSC, Gonzáles AL, Ricós JAD, Real ER. La hospitalización domiciliaria ante los cambios demográficos y nuevos retos de salud. Rev Panam Salud Publica 2002; 11(4):253-261.

5. Camarano AA. Envelhecimento da população brasileira: uma contribuição demográfica: Rio de Janeiro: Ipea; 2002. (Texto para Discussão, no 858).

6. Sousa L, Galante H, Figueiredo D. Qualidade de vida e bem-estar dos idosos: um estudo exploratório na população portuguesa. Rev Saude Publica 2003; 37(3):364-371.

7. Organización Mundial de La Salud. Promoción de la salud: glosario. Genebra: Organización Mundial de La Salud; 1998.

8. Castellón A, Pino S. Calidad de vida en la atención al mayor. Revista Multidisciplinar de Gerontología 2003; 13(3):188-192.

9. Santos SR, Santos IBC, Fernandes MG, Henriques MER. Qualidade de vida do idoso na comunidade: aplicação da escala de Flanagan. Rev Latino-Am Enfermagem 2002; 10(6):757-764.

10. Castellón A, Rubio H, Aleixandre M. Nivel de autoestima en los mayores tras un programa de ocio. Rev Esp de Geriatr Gerontol 1999; 34(4):225-229.

11. Instituto Brasileiro de Geografia e Estatística (IBGE). Sintese de indicadores sociais 2004. Rio de Janeiro: IBGE; 2005.

12. Organización Mundial de La Salud. Envejecimiento activo: un marco político. Rev Esp de Geriatr Gerontol 2002; 37(Supl.2):74-105.

13. Fleck MPA, Louzada S, Xavier M, Chachamovich E, Vieira G, Santos L, Pinzon V. Aplicação da versão em português do instrumento abreviado de avaliação da qualidade de vida "WHOQOL-bref". Rev Saude Publica 2000; 34(2):178-183. 
14. Lima AFBS. Qualidade de vida em pacientes do sexo masculino dependentes de álcool [dissertação]. Porto Alegre: Universidade Federal do Rio Grande do Sul; 2002.

15. Lim L, Fisher J. Use of the 12-item short form (SF12) health survey in an Australian heart and stroke population. Qual Life Res 1999; 8(1/2):1-8.

16. Michelson H, Bolund C, Brandberg Y. Multiple chronic health problems are negatively associated with health-related quality of life (HRQoL) irrespective of age. Qual Life Reses 2001; 9:1093-1104.

17. Jakobsson U, Hallberg IR, Westergren A. Overall and health related quality of life among the oldest old in pain. Qual Life Reses 2004; 1(13):125-136.

18. García LE, Banegas B, Pérez-Regadera G, Cabrera $\mathrm{H}$, Rodríguez-Artalejo F. Social network and health related quality of life in older adults: a populationbased study in Spain. Qual Life Reses 2005; 14(2):440.

19. Statistical Package for the Social Sciences Incorporation. Statistical Package for the Social Sciences for Windows: base system user's guide [programa de computador]. Chicago: SPSS Incorporation; 1992.

20. Instituto de Mayores y Servicios Sociales (IMSER$\mathrm{SO})$. Las personas mayores en España: perfiles - reciprocidad familiar. Madrid: Instituto de Mayores y Servicios Sociales; 1995.

21. Mendes EV. Uma agenda para a saúde. São Paulo: Hucitec; 1996.

22. Cotta Rosângela MM, Mendes Fábio F, Muniz José N. Descentralização das políticas públicas de saúde: "do imaginário ao real". Viçosa, MG: Editora da Universidade Federal de Viçosa; 1998.

23. Pereira DEC. Qualidade de vida na terceira idade e sua relação com o trabalho, no grupo de terceira idade "Amor e Carinho", de Santa Terezinha de Itaipu - PR [dissertação]. Florianópolis: Universidade Federal de Santa Catarina; 2002.

24. Sherbourne CD, Meredith L, Rogers W, Ware J. Social support and stressful life events: age differences in their effects on health related quality of life among chronically ill. Quali Life Res 1992; 1(4):235-246.

25. Gil-Lacruz M, Lanzuela YG, Bravo SM. Tendencias diferenciales de la percepción epidemiológica en función del género. Rev AGATHOS 2005; 5(1):16-23.

26. Hulse G. Alcohol, drugs and much more in later life. Revista ABP 2002; 24(1):34-41.

27. Adlaf E, Smart R. Alcohol use, drug use and wellbeing in older adults in Toronto. Int J Ment Health and Addiction 1995; 20(4):1985-2016.
28. Almeida O, Hulse G, Lawrence D, Flicker L. Smoking as a risk factor for Alzheimer's disease: contrasting evidence from a systematic review of case-control and cohort studies. Addiction 2002; 97(1):15-28.

29. Flemons W, Tsai W. Quality of life consequences of sleep-disordered breathing. J Allergy Clin Immunol 1997; 99(2):S750-S756.

30. Manocchia M, Keller S, Ware J. Sleep problems, health-related quality of life, work functioning and health care utilization among the chronically ill. Qual Life Res 2001; 10(4):331-345.

31. Rosenfeld S. Prevalência, fatores associados e mau uso de medicamentos entre idosos: uma revisão. Cad Saúde Pública 2003; 19(3):717-724.

32. Covinsky K, Wu AW, Landefeld CS, Connors AF, Phillips RS, Tsevat J, Dawson DV, Lynn J, Frotinsky RH. Health status versus quality of life in older patients: does the distinction matter? Am J Med 1999; 106(4):435-440.

33. Thomé B, Dykes A, Hallberg IR. Quality of life in old people with and without cancer. Qual Life Res 2004; 13(3):1067-1080.

34. Sprangers AG, de Regt EB, Andries F, van Agt HM, Bijl RV, de Boer JB, Foets M, Hoeymans N, Jacobs AE, Kempen GI, Miedema HS, Tijhuis MA, de Haes HC. Which chronic conditions are associated with better or poorer quality of life? J Clin Epidemiol 2000; 53(9):895-907.

35. Conselho Nacional de Secretarias Municipais de Saúde (Conasems). Agenda incompleta, diretrizes de gestão e Tese 7 no Conares de maio. Jornal do Conasems 2007; col. 6.

Artigo apresentado em 08/03/2009

Aprovado em 07/08/2009

Versão final apresentada em 05/09/2009 